\title{
Control Method for Autonomous Changing the Number of DC-DC Converters to Improve Efficiency
}

\author{
Masayuki Yamadaya ${ }^{1,2}$, and Hirofumi Matsuo ${ }^{2}$ \\ ${ }^{1}$ Fuji Electric Device Technology Co., Ltd., Matsumoto, Nagano 390-0821, JAPAN \\ ${ }^{2}$ Graduate School of Science and Technology, Nagasaki University, Nagasaki 852-8521, JAPAN \\ E-mail: yamadaya-masayuki@fujielectric.co.jp
}

\begin{abstract}
In this paper, a control method for autonomous changing the number of parallel driven DC-DC converter is proposed. In order to improve efficiency of the parallel driving, the number of parallel driven converters is dynamically changed for the variations of the load current. The changing point of the load current is determined as a function by the number of parallel driven converters and the parameters of the converter. The calculated changing points are usable in practical use with the experimental power loss of the converters.
\end{abstract}

\section{INTRODUCTION}

The load current range of the power supplies becomes higher and wider, and the importance of the power supply system increases. The parallel driving including multi-phase driving are useful to realize high efficiency at heavy load, and also to secure redundancy of the power supply system.

The DC-DC converter modules become very thinner and smaller recently.[1] Appliance of those small converter modules to the parallel driving is expected to realize small size and simple designing of the high efficiency power supply.

However, the parallel driving generally decreases the efficiency at light load as the number of parallel driving increases because of its each fixed loss which does not related to the load current. Therefore, dynamically changing the number of parallel driving is suggested to optimize efficiency for the variations of the load. [2][3]

In order to optimize efficiency by changing the number of parallel driving, it is necessary to find the threshold load current. In this paper, we propose the method to determine the threshold load current simply from the number of parallel driving and the parameters of the converter.

\section{THEORETICAL CONTROL MESHOD}

\section{A. The concept of parallel driving the converters}

Fig. 1 and Fig. 2 show an example of parallel driven buck type DC-DC converter circuit and its efficiency, respectively. $n$ is the number of the parallel driven converters. Input lines and output lines are connected to each other. The parallel driven converters are assumed to have the same performance.

From Fig.2, when the output load current increases, the efficiency generally increases at light load area, and decreases at heavy load area.

In order to realize higher efficiency at heavy load, the parallel driving will be quite useful.

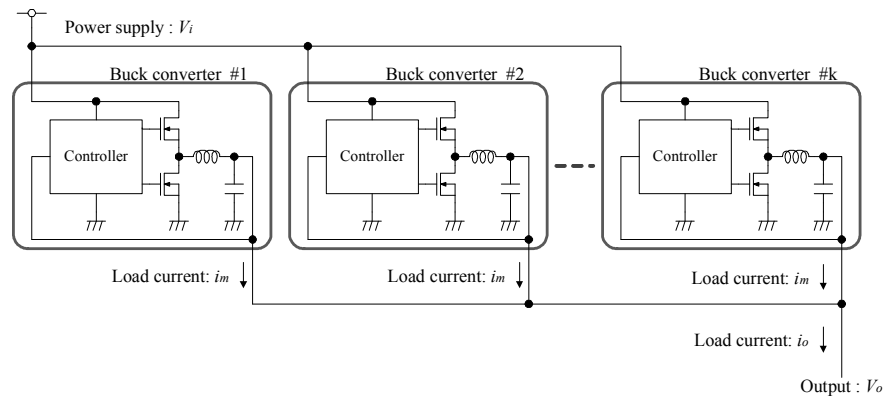

Fig. 1. Buck type DC-DC converter

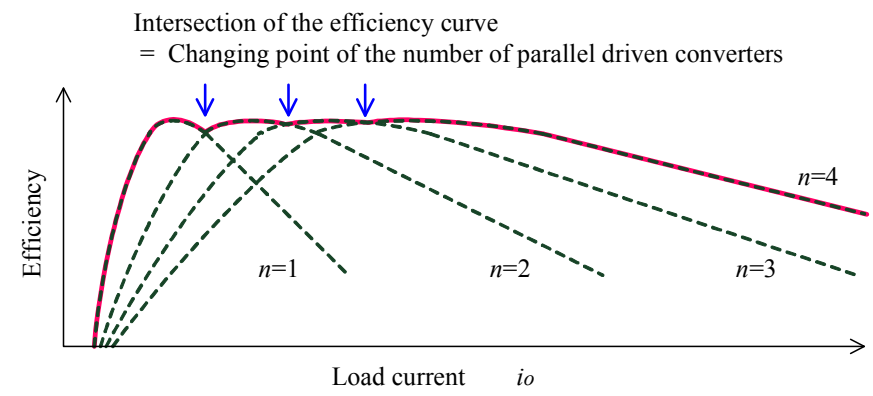

Fig. 2. The efficiency example of a buck type DC-DC converter

If the number of the parallel driven converters is changed at the intersection of the efficiency curve of increased or decreased the number of converters, total efficiency can keeps highest at all times.

Therefore, this paper proposes the method to calculate the intersection of the efficiency curves in the following sections.

\section{B. The elements of the power loss}

First of all, in order to find the intersection of the efficiency curve by calculation, confirming the function which represents the efficiency curve of each buck converter is required.

The power loss of each buck converter in Fig.1 is approximately divided to the following elements.[4]

DC loss of the inductor $\left(P_{L d c}\right)$;

$$
\begin{array}{cl}
P_{L d c}= & R_{L d c} \cdot i_{m}{ }^{2} \\
R_{L d d}: & \text { DC resistance of the inductor } \\
I_{m}: & \text { Output load current of single DC-DC converter }
\end{array}
$$


AC loss of the inductor $\left(P_{L a c}\right)$;

$$
\begin{aligned}
P_{L a c} & =R_{L a c} \cdot I_{L a c}{ }^{2} \\
& =R_{L a c} \cdot\left(\frac{1}{2 \sqrt{3}} \Delta I_{L}\right)^{2} \\
& =\frac{R_{L a c}}{12} \cdot\left(\frac{V_{i}-V_{o}}{L} \cdot \frac{V_{o}}{V_{i}} \cdot \frac{1}{f}\right)^{2}
\end{aligned}
$$

$R_{\text {Lac }}: \quad \mathrm{AC}$ resistance of the inductor

$I_{\text {Lac }}: \quad \mathrm{AC}$ current of the inductor

$$
I_{\text {Lca }}=\frac{1}{2 \sqrt{3}} \Delta I_{L}
$$

$\Delta I_{L}: \quad$ Ripple current of the inductor

$$
\Delta I_{L}=\frac{V_{i}-V_{o}}{L} \cdot \frac{V_{o}}{V_{i}} \cdot \frac{1}{f}
$$

$V_{i}: \quad$ Supply voltage

$V_{o}$ : Output voltage of the DC-DC converter

$L$ : $\quad$ Inductance

$f$ : $\quad$ Switching frequency

Gate charge loss of switching MOSFETs $\left(P_{Q g}\right)$;

$$
P_{Q g}=\left(Q_{g L} \cdot V_{g L}+Q_{g H} \cdot V_{g H}\right) \cdot f
$$

$Q_{g L}: \quad$ Gate charge of low side MOSFET

$Q_{g H}: \quad$ Gate charge of high side MOSFET

$V_{g L}: \quad$ Gate drive voltage of low side MOSFET

$V_{g H}:$ Gate drive voltage of high side MOSFET

Loss of the junction capacitance of the MOSFETs $\left(P_{Q D S}\right)$;

$$
P_{Q D S}=\frac{1}{2}\left(C_{D S L}+C_{D S H}\right) \cdot V_{i}^{2} \cdot f
$$

$C_{D S L}:$ Junction capacitance of low side MOSFET

$C_{D S H}$ : Junction capacitance of high side MOSFET

Conduction loss of the high side MOSFET $\left(P_{\mathrm{ONH}}\right)$;

$$
\begin{aligned}
P_{\text {ONH }} & =\frac{V_{o}}{V_{i}} \cdot R_{\text {ONH }} \cdot \frac{\left(I_{\text {peak }}{ }^{2}+I_{\text {bottom }}{ }^{2}+I_{\text {peak }} \cdot I_{\text {bottom }}\right)}{3} \\
& =\frac{V_{o}}{V_{i}} \cdot R_{O N H} \cdot\left(i_{m}{ }^{2}+\frac{\Delta I_{L}{ }^{2}}{12}\right)
\end{aligned}
$$

$R_{O N H}:$ On resistance of high side MOSFET

$I_{\text {peak }}$ : Peak current of the inductor

$$
I_{\text {peak }}=i_{m}+\frac{\Delta I_{L}}{2}
$$

$I_{\text {bottom }}$ : Bottom current of the inductor

$$
I_{\text {bottom }}=i_{m}-\frac{\Delta I_{L}}{2}
$$

Conduction loss of the low side MOSFET $\left(P_{O N L}\right)$;

$$
\begin{aligned}
P_{O N L} & =\left(1-\frac{V_{o}}{V_{i}}-2 t_{d} \cdot f\right) \cdot R_{O N L} \cdot \frac{\left(I_{\text {peak }}{ }^{2}+I_{\text {bottom }}{ }^{2}+I_{\text {peak }} \cdot I_{\text {bottom }}\right)}{3} \\
& =\left(1-\frac{V_{o}}{V_{i}}-2 t_{d} \cdot f\right) \cdot R_{O N L} \cdot\left(i_{m}{ }^{2}+\frac{\Delta I_{L}{ }^{2}}{12}\right) \\
R_{O N L}: & \text { On resistance of low side MOSFET } \\
t_{d}: & \text { Dead time of high side and low side MOSFET }
\end{aligned}
$$

Conduction loss of the low side body diode $\left(P_{B D I}\right)$;

$$
\begin{aligned}
P_{B D I} & =t_{d} \cdot f \cdot\left(V_{F} \cdot I_{\text {peak }}+V_{F} \cdot I_{\text {bottom }}\right) \\
& =2 t_{d} \cdot f \cdot V_{F} \cdot i_{m}
\end{aligned}
$$

$V_{F}: \quad$ Body diode voltage of low side MOSFET

Turn-on and Turn-off loss of the high side MOSFET $\left(P_{S W}\right)$;

$$
\begin{aligned}
P_{S W}= & \frac{1}{2} \cdot V_{i}\left(I_{\text {peak }} \cdot t_{f}+I_{\text {bottom }} \cdot t_{r}\right) \cdot f \\
= & \frac{1}{2} \cdot V_{i} \cdot f \cdot\left\{\left(t_{f}+t_{r}\right) \cdot i_{m}+\frac{\left(t_{f}-t_{r}\right)}{2} \Delta I_{L}\right\} \\
t_{r}: & \text { Rise time at switching on of high side MOSFET } \\
t_{f}: & \text { Fall time at switching off of high side MOSFET }
\end{aligned}
$$

Power consumption of the control IC $\left(P_{C T R L}\right)$;

$$
\begin{aligned}
P_{C T R L} & =V_{i} \cdot I_{C C} \\
I_{C C}: & \text { Supply current of control IC }
\end{aligned}
$$

The total power loss of each buck converter $P_{L}$ is calculated by the addition of the power loss elements above.

When supply voltage $V_{i}$, output voltage $V_{o}$ and switching frequency $f$ are fixed, the power loss $P_{L d c}$ of (1), $P_{O N H}$ of (7) and $P_{O N L}$ of (8) are the quadratic functions of $i_{m}$, the power loss $P_{B D I}$ of (11) and $P_{S W}$ of (12) are the linear functions of $i_{m}$, and other power losses are constant value.

Therefore, total power loss $P_{L}$ is represented approximately by the quadratic function of load current $i_{m}$.

$$
\begin{aligned}
P_{L}= & \left(P_{L d c}+P_{O N H}+P_{O N L}\right)+\left(P_{B D I}+P_{S W}\right) \\
& +P_{L a c}+P_{Q g}+P_{Q D S}+P_{C T R L} \\
= & a i_{m}{ }^{2}+b i_{m}+c
\end{aligned}
$$

Furthermore, total power loss $P_{L}$ can also be approximated to the quadratic functions of $i_{m}$ by the numerical analysis such as the polynomial regression from the real experimental data of the operated buck converter.

\section{The calculation of the intersection on efficiency curve}

The power loss of the single converter $P_{L}$ is approximates to a quadratic function of the load current $i_{m}$ as follows; .

$$
P_{L}\left(i_{m}\right)=a i_{m}{ }^{2}+b i_{m}+c
$$

When the number of the parallel driven converters is $n$, the total load current $i_{o}$ and the efficiency $\eta_{n}\left(i_{o}\right)$ of parallel driven converters are shown as (17) and (18) respectively.

$$
\begin{aligned}
i_{o} & =n \cdot i_{m} \\
\eta_{n}\left(i_{o}\right) & =\frac{v_{o} i_{o}}{v_{o} i_{o}+n \cdot p_{L}\left(i_{m}\right)}=\frac{v_{o} i_{o}}{\frac{a}{n} i_{o}^{2}+\left(b+v_{o}\right) i_{o}+c n}
\end{aligned}
$$

When the number of the parallel driven converters increase to $n+1$, the total load current $i_{o}$ and the efficiency $\eta_{n+I}\left(i_{o}\right)$ of parallel driven converters are shown as (19) and (20) respectively. 


$$
\begin{aligned}
i_{o}=(n+1) \cdot i_{m} & \\
\eta_{n+1}\left(i_{o}\right) & =\frac{v_{o} i_{o}}{v_{o} i_{o}+(n+1) \cdot p_{L}\left(i_{m}\right)} \\
= & \frac{v_{o} i_{o}}{\frac{a}{n+1} i_{o}{ }^{2}+\left(b+v_{o}\right) i_{o}+c(n+1)}
\end{aligned}
$$

From Fig.2, the ideal changing point is the intersection of the efficiency curves (18) and (20). Therefore, the intersection load current $i_{o}=I_{t h \_n}$ of the efficiency curve is calculated from the functions (18) and (20) as follows.

$$
\begin{aligned}
& \eta_{n}\left(I_{t h_{-} n}\right)=\eta_{n+1}\left(I_{t h_{-} n}\right) \\
& \frac{v_{o} I_{t h_{-} n}}{\frac{a}{n} I_{t h_{-} n}{ }^{2}+\left(b+v_{o}\right) I_{t h_{-} n}+c n} \\
& =\frac{v_{o} I_{t h_{-} n}}{\frac{a}{n+1} I_{t h_{-} n}{ }^{2}+\left(b+v_{o}\right) I_{t h \_n}+c(n+1)} \\
& I_{t h_{-} n}=\sqrt{\frac{c}{a} n(n+1)}
\end{aligned}
$$

Therefore the intersection load current of each converter $I_{m t h \_n}$ is calculated as follows;

$$
I_{m t h_{-} n}=\frac{I_{t h_{-} n}}{n}=\sqrt{\frac{c}{a} \frac{n+1}{n}}
$$

Equation (24) means that the intersection load current of each converter is determined by the coefficient of the quadratic function $a$ and $c$ which represents the efficiency curve as (16), and the number of parallel driven converters $n$.

Furthermore, since the coefficient $a$ and $c$ are fixed value, the intersection load currents of (23) and (24) is only the function of $n$.

\section{Detection circuit}

From (24), the intersections of the efficiency curves are calculated by comparing the sensed load current $i_{m}$ with the reference voltage which related to $n$.

Therefore, the detection circuit of the efficiency curve intersection can be composed with the comparator which compares the sensed load current $i_{m}$ and the reference voltage $V_{R E F}$ as shown in Fig.3.

In Fig.3, the coefficient " $\alpha$ " is multiplied to both of the sensed current " $i_{m}{ }^{2}$ " and the reference voltage " $c / a(n+1) / n$ " in order to operate the comparator in its common-mode range.

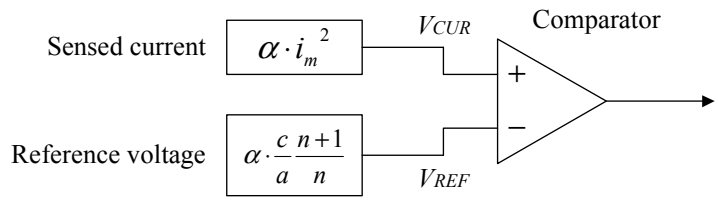

Fig.3 The detection circuit of the changing point

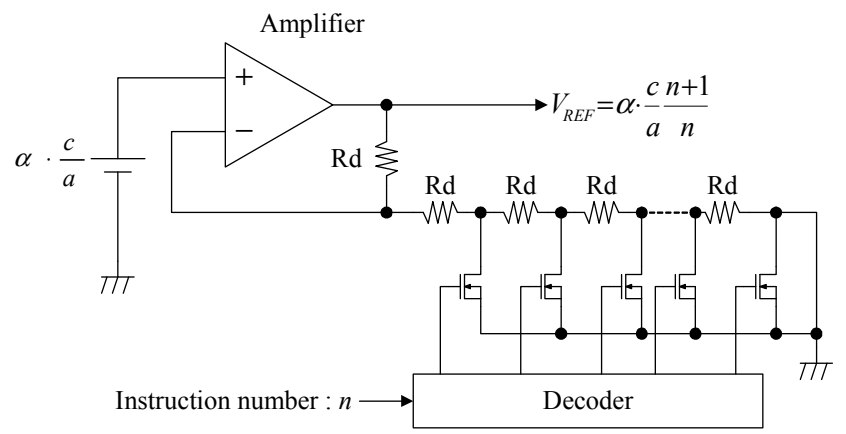

Fig.4 Reference voltage circuit

The reference voltage $V_{R E F}$ can be created by the simple regulator circuit as shown in Fig.4. $V_{R E F}$ is determined by the number of the resistance $R_{d}$ in voltage divider. The number of resistance $R_{d}$ is changed with the instructed number $n$ which is the number of parallel driven converters.

Current sense circuit which outputs the voltage $V_{C U R}\left(=\alpha i_{m}{ }^{2}\right)$ should be composed with the applied multipliers. In this paper, the explanation of the current sense and multiplier circuits are omitted.

\section{E. Verification of the proposed method}

In order to verify the proposed method to find the intersection of the efficiency curves, we compared the power loss curves and the efficiency curves with the experimental result, theoretical quadratic function by the equations from (1) to (15), and approximated quadratic function by the polynomial regression of the experimental result.

The experimental power loss and the efficiency are evaluated by using trial products of $3 \mathrm{~A}$ built-in MOSFET synchronous buck converter IC which is shown as Fig.5.

The circuit design parameters of the converter parts and ICs are shown in Table.1. Therefore, equations from (1) to (15) can be calculated by the parameters in Table. 1

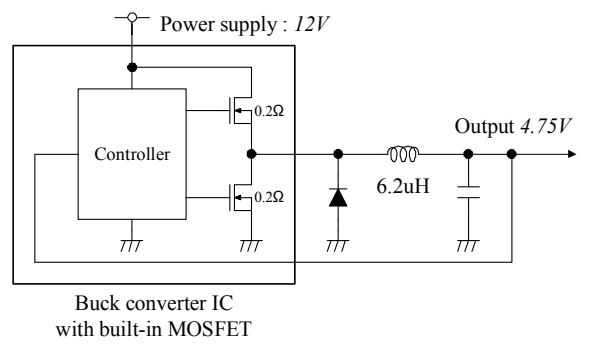

Fig.5 Experimental buck converter

TABLE 1. CIRCUIT DESIGNE PARAMETERS

\begin{tabular}{|c|c|c|c|c|c|c|c|c|}
\hline Item & Val. & Unit & Item & Val. & Unit & Item & Val. & Unit \\
\hline$V_{i}$ & 12 & $\mathrm{~V}$ & $Q_{g H}$ & 2.82 & $\mathrm{nC}$ & $t_{r}$ & 10 & $\mathrm{~ns}$ \\
\hline$V_{o}$ & 4.75 & $\mathrm{~V}$ & $V_{g L}$ & 5.0 & $\mathrm{~V}$ & $t_{f}$ & 20 & $\mathrm{~ns}$ \\
\hline$f$ & 460 & $\mathrm{kHz}$ & $V_{g H}$ & 5.0 & $\mathrm{~V}$ & $t_{d}$ & 10 & $\mathrm{~ns}$ \\
\hline$L$ & 6.2 & $\mu \mathrm{H}$ & $C_{D S L}$ & 360 & $\mathrm{pF}$ & $V_{F}$ & 0.45 & $\mathrm{~V}$ \\
\hline$R_{d c}$ & 16 & $\mathrm{~m} \Omega$ & $C_{D S H}$ & 360 & $\mathrm{pF}$ & $I_{C C}$ & 0.5 & $\mathrm{~mA}$ \\
\hline$R y$ & 0.5 & $\Omega$ & $R_{O N L}$ & 0.2 & $\Omega$ & & & \\
\hline$Q_{g L}$ & 2.82 & $\mathrm{nC}$ & $R_{O N H}$ & 0.2 & $\Omega$ & & & \\
\hline
\end{tabular}


The power loss $P_{L}$ is calculated from (14) and the Table.1 as follows;

$$
P_{L}\left(i_{m}\right)=0.214 i_{m}{ }^{2}+0.0745 i_{m}+0.122
$$

The experimental power loss of the buck converter is shown in Fig.6. It also shows the theoretical power loss which is calculated from the function of (25). The theoretical power loss curve is almost as same as the experimental power loss curve. Therefore the theoretical function (25) has few errors at modeling is confirmed.

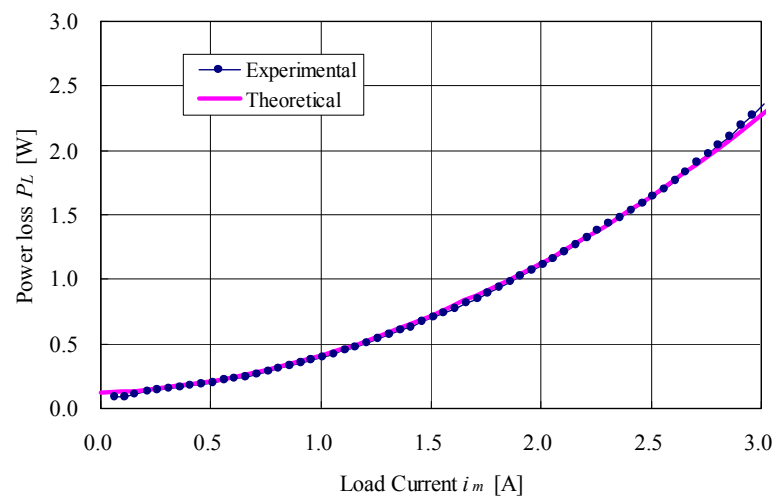

Fig.6 Power loss curve of the buck converter

The experimental power loss curve in Fig.6 can be approximated by using polynomial regression.

The approximated power loss function is shown as follows;

$$
P_{L}\left(i_{m}\right)=0.234 i_{m}{ }^{2}+0.0311 i_{m}+0.126
$$

Therefore, the each coefficient $a, b$ and $c$ of quadratic function (16) are shown in Table.2.

TABLE 2. CODEFFICIENTS OF QUADRATIC FUNCTION

\begin{tabular}{|l|c|c|c|}
\hline & $a$ & $b$ & $C$ \\
\hline Theoretical function & 0.214 & 0.0745 & 0.122 \\
\hline Approximated function & 0.234 & 0.0311 & 0.126 \\
\hline
\end{tabular}

The efficiency curves are shown in Fig.8. It compares with the experimental result, theoretical function of (25), and approximated function of (26).

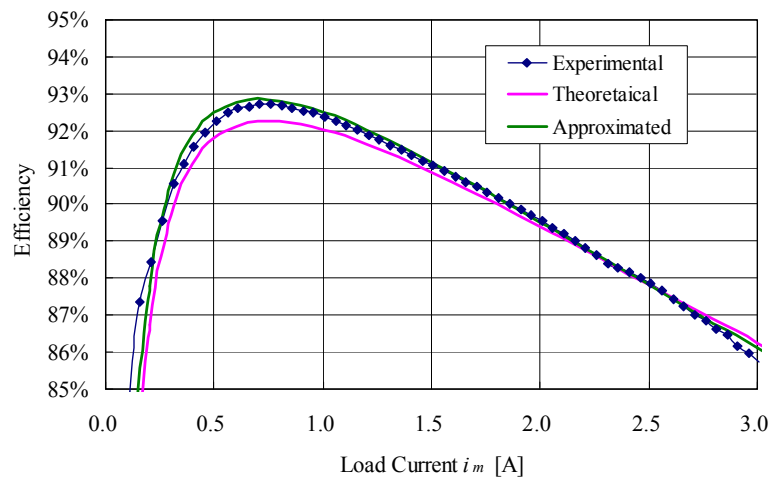

Fig.8 Efficiency curve of the buck converter
It is realized the theoretical curve and the approximated curve include some errors from the comparison to the experimental result.

The intersections of efficiency curves which are calculated as (23), (24) and Fig.3 are shown in Table.3.

TABLE 3. THE INTERSECTION OF THE EFFICIENCY

\begin{tabular}{|c|c|c|c|c|}
\hline \multirow{3}{*}{$n$} & \multicolumn{2}{|c|}{ Theoretical } & \multicolumn{2}{c|}{ Approximated } \\
\cline { 2 - 5 } & \multicolumn{2}{|c|}{$\begin{array}{c}c / a=0.570 \\
\alpha=1.0\end{array}$} & \multicolumn{2}{c|}{$\begin{array}{c}c / a=0.502 \\
\alpha=1.0\end{array}$} \\
\cline { 2 - 5 } & $V_{\text {REF }}[\mathrm{V}]$ & $I_{\text {th_n }}[\mathrm{A}]$ & $V_{R E F}[\mathrm{~V}]$ & $I_{\text {th_n}}[\mathrm{A}]$ \\
\hline 1 & 1.140 & 1.068 & 1.004 & 1.002 \\
\hline 2 & 0.855 & 1.849 & 0.753 & 1.736 \\
\hline 3 & 0.760 & 2.615 & 0.669 & 2.454 \\
\hline 4 & 0.713 & 3.376 & 0.628 & 3.169 \\
\hline
\end{tabular}

Fig.7 shows the experimental efficiency curves with the calculated intersections which are shown in Table.3. The calculated intersections are almost as same as the intersection of the experimental curves.

Each difference between the intersections of the experimental curves and the calculated intersections are shown in Table.4.

The error of the intersection current $\Delta I_{\text {th } n}$ seems slightly larger. However, the intersection efficiency errors $\Delta \eta_{n}\left(I_{t h} n\right)$ of both theoretical result and approximated result are less than $0.2 \%$, the calculated intersection are usable enough in practical use.

This result shows the change of the efficiency around the intersection is not large. Therefore a slight decrease of the calculated intersection current accuracy may be allowed.

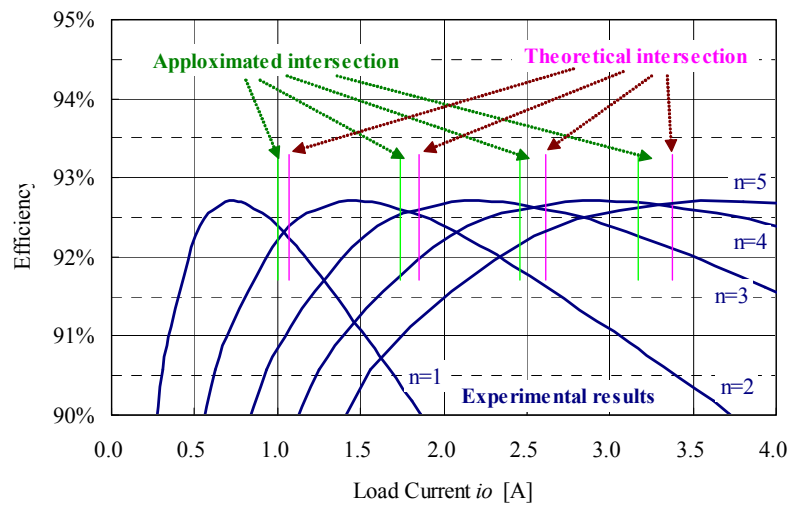

Fig.7 Efficiency curve

TABLE 4. THE DIFFERENCES FROM THE IDEAL INTERSECTION

\begin{tabular}{|c|c|c|c|c|}
\hline \multirow{2}{*}{$n$} & \multicolumn{2}{|c|}{ Theoretical } & \multicolumn{2}{c|}{ Approximated } \\
\cline { 2 - 5 } & $\Delta I_{t h \_n}$ & $\Delta \eta_{n}\left(I_{t h \_n}\right)$ & $\Delta I_{t h \_n}$ & $\Delta \eta_{n}\left(I_{t h \_n}\right)$ \\
\hline 1 & $2.84 \%$ & $-0.14 \%$ & $-3.51 \%$ & $-0.15 \%$ \\
\hline 2 & $3.09 \%$ & $-0.08 \%$ & $-3.21 \%$ & $-0.08 \%$ \\
\hline 3 & $2.59 \%$ & $-0.05 \%$ & $-3.73 \%$ & $-0.08 \%$ \\
\hline 4 & $2.43 \%$ & $-0.04 \%$ & $-3.85 \%$ & $-0.07 \%$ \\
\hline
\end{tabular}




\section{CONCLUSION}

We proposed the new method to change the number of parallel driven buck type DC-DC converters to improve efficiency. If you can represent the power loss of the converter to the quadratic function, the threshold load current of changing the number of converters can be represented simply from the number of parallel driven converters and the parameters of the converter.

We used the buck converter to explain the method in this paper. However this method is usable not only buck converters, but also any converters which the power loss of the converter can be represented to the quadratic function.

We will examine to apply this method with variable input and output voltage, frequency and temperature in near future.

\section{REFERENCES}

[1] Kouhei Yamada, Satoshi Sugahara, Low Quiescent Current and Small Size DC-DC Converters for Portable Appliances, Technical Meeting on Electronic Circuits IEEJ ECT-06-91 (Oct. 2006)

[2] P.Zumel, C.Fernandez, A.dc Castro,O.Garcia, "Efficiency improvement in multiphase converter by changing dynamically the number of phases," PESC'06, ThA2-3, pp. 2845-2850

[3] Jaber A. Abu Qahouq and Lilly Huang, "Power Converter with Gradient Power Architecture and Non-Uniform Current Sharing," INTELEC2006, 23-3, pp. 349-356

[4] KATAYAMA Yasushi, EDO Masaharu, DENTA Toshio, KAWASHIMA Tetsuya, NINOMIYA Tamotsu, "Optimum Design of CMOS DC-DC Converter for Mobile Applications" [in Japanese], IEEJ Transactions on Industry Applications 124(10), 1043-1052,20041001 